\title{
Efeitos da luz em sistemas biológicos
}

\section{Effects of light on biological systems}

\author{
Ivan Frederico Lupiano Dias ${ }^{1}$; Cláudia Patrícia Cardoso Martins Siqueira²; \\ Dari de Oliveira Toginho Filho ${ }^{3}$; José Leonil Duarte ${ }^{1}$; Edson Laureto'; \\ Franciele Mendes de Lima ${ }^{4}$; Francisco Pereira Silva ${ }^{5}$; Valdênea Aparecida \\ Bordinassi de Castro $^{6}$; Roberto Kiyoshi Kashimoto ${ }^{7}$; Henrique Durante ${ }^{7}$
}

\section{Resumo}

Embora haja atualmente um extenso emprego de fontes de luz como LASERs (Light Amplification by Stimulated Emission of Radiation) e LEDs (Light Emitting Diodes) em diversas técnicas terapêuticas, as bases científicas de seus efeitos sobre sistemas biológicos ainda é relativamente desconhecida pela comunidade acadêmica. Neste estudo é apresentada uma breve revisão sobre os efeitos da luz em sistemas biológicos. O emprego de fontes de luz de baixa potência, como diodos emissores de luzLEDs, pode propiciar um recurso terapêutico opcional aos convencionais ou ser utilizado em conjunto com estes, com a vantagem do baixo custo e comprovada eficiência no tratamento de úlceras e outras enfermidades.

Palavras-chave: Propriedades óticas. Efeitos biológicos. Diodo emissor de luz. Led.

\begin{abstract}
Although nowadays light sources such as LASERs (Light Amplification by Stimulated Emission of Radiation) and LEDs (Light Emitting Diodes) are largely used in several therapeutic areas, the scientific basis of their effects on biological systems still is relatively unknown by the academic community. In this study, a brief revision about effects of light on biological systems is presented. Low power light sources such as LEDs may be used as an alternative therapeutic method or be used associated to the conventional methods, having the advantage of being a low cost method and of proven efficiency in the treatment of ulcers and other diseases.
\end{abstract}

Key words: Optical properties. Biological effects. Light emitting diodes. Led

1 Docente do Departamento de Física da Universidade Estadual de Londrina - UEL; idias@uel.br

Docente do Departamento de Fisioterapia da Universidade Estadual de Londrina - UEL; claudia@uel.br

Físico do Departamento de Física da Universidade Estadual de Londrina - UEL; darit@uel.br

${ }^{4}$ Graduanda do curso de Física da - UEL; fran_mendes_lima@hotmail.com

5 Docente do Departamento de Clínica Cirúrgica da UEL; fpereira@sercomtel.com.br

6 Enfermeira do H.C. da Universidade Estadual de Londrina - UEL valdenea@sercomtel.com.br

7 Residentes do curso de Fisioterapia da UEL; hedurante@yahoo.com ; robertofisiouel@yahoo.com.br 


\section{Introdução}

O estudo de fenômenos luminosos, da verdadeira natureza da luz, e o desenvolvimento de sua aplicação ocorre desde a antiguidade. Estudos de fenômenos elétricos e magnéticos e de sua natureza também encontram registro desde o início da história das civilizações. Até o final do século XVII, início do século XVIII, preponderou o entendimento estabelecido por Newton de que a luz era composta por feixes de partículas. No início do século, contudo, Young, ao fazer a luz incidir sobre fendas, mostrou experimentalmente o surgimento de franjas de interferência resultado que somente poderia ser explicado caso a luz tivesse um comportamento ondulatório. A relação entre fenômenos elétricos e magnéticos (eletromagnetismo) e a luz ocorreu somente em meados do século XVIII, com os trabalhos de Michael Faraday, ao analisar os efeitos de campos magnéticos fortes na direção de polarização de um feixe luminoso. As bases matemáticas e o entendimento da luz como um fenômeno ondulatório se deu graças aos trabalhos de Maxwell (James Clerk Maxwell) no final do mesmo século.

Os conceitos de partícula e onda só fazem sentido dentro do escopo da Física Clássica (determinística). O trabalho de Planck, em 1900, ao introduzir a noção de quantas de energia para explicar o comportamento da radiação de um corpo negro levou ao entendimento que a radiação eletromagnética poderia, em determinadas condições experimentais, apresentar características corpusculares. Um exemplo disto é o efeito fotoelétrico, discutido por Einstein em 1905. De acordo com a teoria corpuscular, a luz consiste de partículas ou quantas com energia proporcional à freqüência $v$ e energia igual a $\mathrm{E}=h v$, onde $h$ é a constante de Planck. De 1900 a 1930, com os trabalhos de Bohr, de Broglie e Schrödinger, entre outros, foi introduzida a Física Quântica, onde a noção onda/partícula deixou de ter sentido. No contexto da Física Quântica a luz não é partícula nem onda, não tendo sentido, na verdade, essa pergunta dado o caráter probabilístico dos entes quânticos. A natureza da matéria se expressa em fenômenos ocorrendo em escala de dimensões atômicas de tal modo que, dependendo da experiência, o objeto apresenta características de onda ou partícula noções de Física clássica que são empregadas de modo a se ter uma noção mais intuitiva dos fenômenos observados.

O desenvolvimento da mecânica quântica propiciou o surgimento da espectroscopia molecular com o emprego da luz para análise de estruturas em nível molecular e a possibilidade de sua utilização para o estudo e análise de sistemas biológicos complexos. O forte desenvolvimento tecnológico, a partir de meados do século XX, com o desenvolvimento de dispositivos como o LASER em 1962, por exemplo, abriu possibilidades para a aplicação da luz em vários campos do conhecimento humano inclusive na medicina. As bases para a compreensão dos mecanismos moleculares associados aos efeitos da luz sobre as células foram estabelecidas, entretanto, somente na década de 80 .

Atualmente uma série de técnicas empregando propriedades da luz como, reflexão, espalhamento, interferência, coerência, entre outras, estão sendo amplamente utilizadas em diagnósticos biomédicos, desenvolvimento de técnicas não invasivas e no tratamento de enfermidades em vários ramos da medicina, como dermatologia, oftalmologia, urologia, oncologia entre outros. Uma excelente revisão sobre o tema pode ser encontrada no texto Biomedical Photonics Handbook, editado por Tuan Vo-Dinh, em 2003 (VO-DINH, 2003). No Brasil vários grupos de pesquisa atuam neste campo e verifica-se a incorporação de técnicas empregando luz em uma série de tratamentos de rotina e diagnóstico em clínicas, laboratórios e hospitais. A pesquisa neste campo, entretanto, ainda está em seu início.

Entre as possíveis aplicações da luz, seu emprego no tratamento de úlceras, com a utilização de LASERs de baixa potência ou LEDs, já encontra vários registros na literatura e pode propiciar um 
recurso terapêutico opcional aos convencionais ou ser utilizado em conjunto com estes, com a vantagem do baixo custo e comprovada eficiência (MARQUES, 2004), (CORAZZA, 2005). Neste trabalho é apresentada uma revisão sobre os efeitos da luz em sistemas biológicos.

\section{Luz em sistemas biológicos}

Efeitos da luz sobre sistemas biológicos são conhecidos há bastante tempo. De acordo com o trabalho de T. Karu (1987), existem registros sobre os efeitos da radiação eletromagnética, em vários comprimentos de onda na região do visível $(400 \mathrm{~nm}$ a $700 \mathrm{~nm}$ ), sobre organismos e células já na metade final do século 19 (GORBACHEWICH, 1882; SCHNETZLER, 1874; FUBINI, 1879; PONZA, 1876). Ainda de acordo com a mesma autora, na década de 20 e 30 vários estudos sobre os efeitos da luz com comprimentos de onda nos extremos do espectro visível (vermelho e azul) sobre vários processos bioquímicos foram obtidos (VERDONK, 1937; POLIAKOV; MARGOLIN; FEDDER, 1935; KÜSTNER, 1931). Foi no início da década de 60 porém, com a produção do primeiro dispositivo com amplificação da luz por emissão estimulada de radiação, o LASER de rubi, por Theodore H. Maiman (MAIMANN, 1960) e principalmente a fabricação do primeiro LASER de Hélio - Neônio (He-Ne), por Javan, Bennet e Herriot (1961), que a aplicação da luz em várias áreas do conhecimento humano (e em especial na medicina) ganhou intensidade.

A invenção do LASER tornou possível obter luz com grande potência e em comprimentos de onda bem definidos (monocromaticidade). Isto facilitou sua aplicação em vários campos do conhecimento e a determinação dos efeitos biológicos da radiação em regiões muito específicas de comprimentos de onda (ou energia ou freqüência) do espectro de radiação eletromagnético. No início da década de 70, Mester e colaboradores (Mester et al, 1971; Mester et al, 1972), em Budapeste, Hungria, desenvolveram um trabalho pioneiro na aplicação de LASERs de rubi in vitro e in vivo em úlceras crônicas obtendo resultados efetivos no processo de cicatrização. $\mathrm{O}$ LASER de He-Ne, com emissão em 632,8 nm, foi o primeiro LASER disponível comercialmente, o que levou ao forte desenvolvimento do estudo dos efeitos da luz com este comprimento de onda (cor vermelha) em aplicações médicas.

No final da década de 60, início da década de 70, surgiram os primeiros LASERs de materiais semicondutores, abrindo possibilidades ao emprego de LASERs relativamente baratos, de fácil manuseio e ao desenvolvimento de terapia com LASERs de baixa potência no que é conhecido atualmente pela sigla - LLLT (low level laser therapy ou low power laser therapy). Os primeiros lasers semicondutores eram fabricados com GaAs (arseneto de gálio) ou ligas mais sofisticadas deste material como AlGaAs geralmente com emissão na região do infravermelho $(780 \mathrm{~nm}$ a $904 \mathrm{~nm})$. Atualmente, graças aos avanços nesta área, estruturas compostas por diferentes materiais semicondutores como InGaAsP, AlGaInAs, GaN, AlGaN etc. permitem a construção de LASERs de semicondutores com emissão em todo o espectro visível (SCHUBERT, 2006).

A grande profusão de trabalhos, com o emprego de parâmetros pouco sistematizados [relacionados ao comprimento de onda, à largura espectral, à intensidade, às condições dos pacientes, às condições da amostra/objeto de estudo etc.], levou à contradições nos resultados (KARU, 1987) e a um grande ceticismo por parte da comunidade científica (físicos, biólogos, químicos) e comunidade médica sobre a aplicação prática da luz. Além disso, até o início da década de 80, desconheciam-se os mecanismos de ação da radiação eletromagnética em nível molecular e celular (KARU, 1987; KARU, 2003).

T. Karu, em uma série de trabalhos científicos realizados ao longo da década de 80 (KARU et al., 1982a; KARU et al., 1983c; KARU et al., 1983a) estabeleceu as bases para a compreensão dos 
mecanismos moleculares associados aos efeitos da luz sobre as células. Sabe-se atualmente que existe um mecanismo foto-biológico universal da ação da luz na cadeia respiratória de células eucarióticas e procarióticas com enzimas terminais da cadeia respiratória, na mitocôndria, atuando como fotoaceitadores (KARU; KOLYAKOV, 2005). As respostas celulares surgem em reações primárias na cadeia respiratória e secundárias no citoplasma e no núcleo das células. Outro resultado de T. Karu foi a evidência de que a resposta celular à fotoestimulação não está associada a propriedades específicas da luz LASER, como a coerência. Isto abriu espaço para o trabalho com fontes emissoras de luz não coerentes como os diodos emissores de luz - LEDs. Estes dispositivos são mais baratos, de maior facilidade de manuseio, e operam com correntes elétricas relativamente baixas em comparação aos LASERs (SCHUBERT, 2006).

LASERs podem ser fabricados com diferentes tipos de meios ativos (onde ocorrem os processos de geração da luz) como materiais sólidos como rubi, $\mathrm{Nd}$ :YAG, semicondutores etc. ou gasosos como $\mathrm{CO}_{2}$ , Ar, He-Ne etc. ou líquidos como corantes. No caso de meio ativo composto de materiais semicondutores ambos, um LED ou um LASER, são estruturas que podem ser caracterizadas como diodos emissores de luz. O LASER, entretanto apresenta sofisticação estrutural com a presença de uma cavidade óptica ressonante. Isto é, a luz, que é gerada pela passagem de corrente elétrica no dispositivo é emitida de forma estimulada e apresenta características como a coerência: a radiação eletromagnética oriunda da recombinação de cargas elétricas dentro do material é emitida com a mesma diferença de fase e com a mesma orientação. Isto permite grande concentração do feixe de luz (colimação) ao longo de uma direção e a emissão em um comprimento de onda muito específico. T. Karu observou, entretanto, que esta propriedade de coerência é perdida na interação da luz com o tecido biológico, não sendo, portanto uma condição essencial para o processo de foto estimulação (KARU, 1987).
O emprego de LEDs e/ou LASERs de baixa potência com finalidades terapêuticas vem sendo muito ampliado ao longo dos últimos anos com farta literatura disponível em sites (MEDICAL COLLEGE OF WINSCONSIN, 2008; NASA, 2008) e em diversas revistas científicas ${ }^{8}$. A foto estimulação causada pela irradiação por LEDs ou LASERs de baixa potência apresenta uma série de resultados tais como o aumento da taxa de proliferação de células (KARU, 1987), o aumento da taxa de produção de fibroblastos e da síntese de colágenos (TAKAHASHI, 1992; VINCK et al., 2003), aumento da taxa de síntese de RNA e DNA (KARU, 1987; KARU; KOLYAKOV, 2005), síntese de ATP (YU et al, 1997), aumento da vascularização (CONLAN; RAPLEY; COBB, 1996), variações na condução nervosa (VINCK et al., 2005), etc.

A aplicação do LED como recurso terapêutico vem se ampliando na última década. Estudos desenvolvidos pela NASA (National Aero Space Agency) nos Estados Unidos demonstraram fortes evidências de que a eficiência dos LEDs em processos de foto estimulação celular é semelhante à dos LASERs de baixa potência - terapia LLLT (VINCK et al., 2003). Testes clínicos com aplicação de LEDs em cicatrização de úlceras em seres humanos já foram liberados pela FDA (Food Drug Administration) norte americana (WHELAN et al., 2001). Trabalhos recentes desenvolvidos no Brasil demonstram a eficiência deste recurso para o tratamento de diversos tipos de úlceras (MARQUES, 2004), (CORAZZA, 2005).

\section{Reações fotobiológicas}

A reação fotobiológica à aplicação da luz em determinados comprimentos de onda é fornecida por um espectro de ação. Um espectro de ação é um gráfico de fotoresposta, por exemplo, entre outros, da taxa de síntese de DNA ou RNA em células HeLa ou o crescimento de Escheria Coli em função do

\footnotetext{
Photomedicine and Laser Surgery, Lasers in Medical Science, Photochemical Photobiology.
} 
comprimento de onda $\lambda$, da freqüência $v$, ou da energia hv. As reações fotobiológicas se devem à absorção por uma molécula fotoaceitadora da energia da luz incidente. Um espectro de ação deve ser, portanto, idealmente similar ao espectro de absorção da molécula que absorve a luz. Os primeiros espectros de ação foram obtidos em meados da década de 80 , por T. Karu e diversos colaboradores, em uma série de trabalhos sobre a síntese de DNA e RNA (KARU et al., 1983a; KARU et al., 1983b), crescimento de Escheria Coli (KARU et al., 1983c; KARU et al., 1984) e síntese de proteína por "yeasts" (KARU, 1984) com aplicação da luz na região do visível.

Na Figura 1 apresentamos três espectros de ação considerando a taxas de síntese de $\operatorname{DNA}(\boldsymbol{\Delta})$, a taxa de síntese de RNA (•), a \% de adesão de células HeLa na fase exponencial (ロ) e um espectro de absorção de monocamadas de células de HeLa (-) secas a ar na região de comprimentos de onda do vermelho ao infravermelho próximo $(550 \mathrm{~nm}$ a 900 $\mathrm{nm})$. Esta figura é readaptada de figura publicada na literatura por T. Karu [Figura 48.4 - Handbook]. As células HeLa caracterizam-se por um grupo de células cancerosas doadas involuntariamente por Henrietta Lacks (1920-1951). Henrietta Lacks era portadora de um câncer de colo uterino que produzia metástases anormalmente rápidas. Após sua morte, estas células continuaram a ser cultivadas em laboratórios e auxiliam em várias pesquisas como vacinas para a poliomielite, câncer, AIDS, efeitos da radiação e mapeamento genético.

Um primeiro dado importante a observar é que as posições dos picos dos espectros de ação são praticamente as mesmas respeitando-se os limites do erro experimental. Isto é muito interessante uma vez que os processos analisados ocorrem em diferentes regiões da célula, no núcleo e membrana plasmática. Portanto é possível concluir que o fotoaceitador primário seja o mesmo. Uma outra conclusão é que devem existir diferentes caminhos de sinalização (signaling pathways) dentro da célula entre o fotoaceitador, o núcleo e a membrana celular. Acredita-se atualmente que existe um mecanismo universal fotobiológico da ação da luz sobre a cadeia respiratória em células eucarióticas e procarióticas. Os fotoaceitadores são enzimas terminais da cadeia respiratória: a citocroma c oxidase e o citocroma bd em células procarióticas de Escheria Coli, no caso da radiação na região do vermelho ao infravermelho próximo NIR (Near Infra Red). Na região do azul ao violeta outros fotoaceitadores como flavoproteínas em outras etapas da cadeia respiratória foram identificados. Diferenças entre os espectros na região de $750 \mathrm{~nm}$ são provávelmente devidas ao espalhamento de luz e inomogeneidades dos tecidos em multicamadas (KARU, 2005). É importante salientar que condições experimentais podem interferir no estado de oxidação/redução do citocroma c oxidase que só é um fotoaceitador primário quando está em forma intermediária (parcialmente reduzido, por exemplo) (KARU, 2003).

Os mecanismos de ação da luz após a absorção se caracterizam em primários e secundários. Os mecanismos primários ainda não foram bem estabelecidos com uma série de hipóteses associadas à geração de oxigênio singlete ${ }^{1} \mathrm{O}_{2}$ (KARU, 1989), alteração das propriedades do estado excitado redox dos centros $\mathrm{Cu}_{\mathrm{A}}$ e $\mathrm{Cu}_{\mathrm{B}}$ ou heme a e heme $a_{3}$ (KARU, 1988), presença de óxido nítrico (NO) (BROWN, 1999), aquecimento local transiente (KARU et al., 1991) ou aumento da produção da anions superóxidos (KARU; ANDREICHUK; RUABYKH, 1993). De acordo com Karu (2003) os diferentes mecanismos podem levar a resultados similares estimulando o estado redox da mitocôndria na direção de maior oxidação. Discute-se ainda, contudo, quais mecanismos são decisivos em uma dada situação, pois fatores como a dose de luz e a intensidade usada podem favorecer a predominância de um ou outro (KARU, 2003). Os mecanismos secundários são os responsáveis pela conexão entre a resposta à ação da luz pelos fotoaceitadores localizados na mitocôndria e os mecanismos de síntese de DNA e RNA localizados no núcleo ou outros fenômenos em outros componentes da célula, como por 
exemplo, a adesão da membrana. Existem diferentes processos de regulação associados ao controle dos fotoaceitadores sobre o nível de ATP intracelular, fatores de transcrição celular sensíveis ao estado redox ou cascatas homeostáticas de sinalização celular do citoplasma através da membrana da célula para o núcleo (KARU, 2003). Estes mecanismos apresentam dependência de vários parâmetros como a dose de irradiação, o comprimento de onda, o modo de operação da excitação luminosa (pulsado/contínuo) e a intensidade de excitação. Foi observado que o estado geral redox da célula e seu pH no momento da irradiação podem levar a respostas mais fortes ou mais fracas das células irradiadas, isto é, a resposta à excitação, principalmente a de baixa potência, depende das condições iniciais da célula. Como estas condições dependem de uma série de fatores e variam de indivíduo para indivíduo a resposta aos efeitos da irradiação ainda necessitam de uma análise mais sistemática principalmente para estabelecer correlações quantitativas.

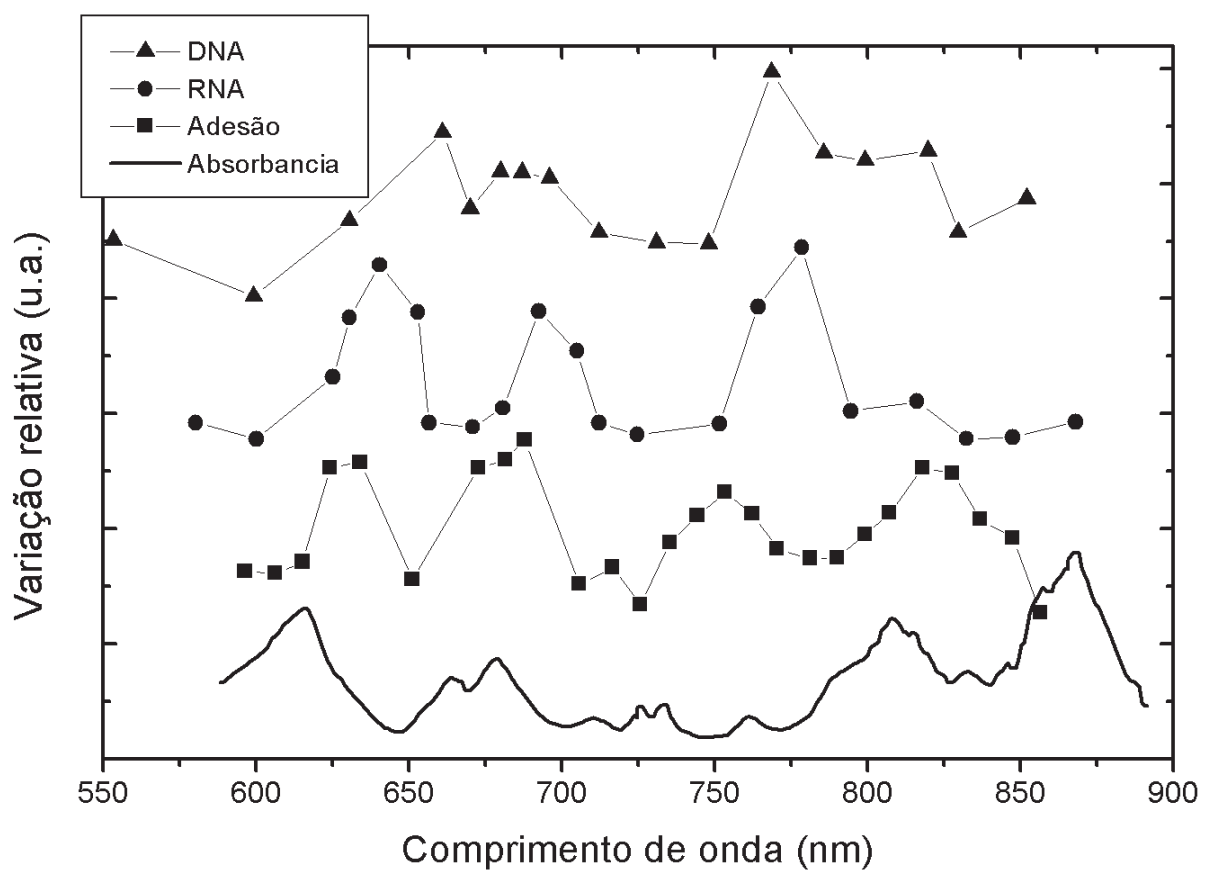

Figura 1. Taxas de síntese de DNA $(\boldsymbol{\Delta})$, RNA $(\bullet)$ e \% de adesão de células na fase exponencial (ロ) de células HeLa e um espectro de absorção de monocamadas de células de HeLa (-) secas a ar na região de comprimentos de onda do vermelho ao infravermelho próximo $(550 \mathrm{~nm}$ a $900 \mathrm{~nm}$ ) readaptada da figura publicada na literatura por T. Karu [Figura 48.4 - Handbook]. As unidades são dadas em DNA - $\mathrm{H}^{3} \mathrm{DMPx} 10^{3} / 4 \times 10^{5}$ células, RNA - $\mathrm{C}^{14}$ DMPx $10^{3} / 2,5 \times 10^{5}$ células, taxa de adesão - \% e absorbância - \%. Os gráficos estão normalizados pelo valor obtido no comprimento de onda de $550 \mathrm{~nm}$. 


\section{Conclusões}

A compreensão sobre os efeitos da luz em sistemas biológicos, em especial a luz com baixa intensidade, encontra-se ainda em fase inicial, mas já existe vasta bibliografia mostrando ser este um recurso terapêutico com resultados interessantes em vários ramos da medicina. Esperamos que este breve resumo possa contribuir para despertar o interesse de pesquisadores de diferentes áreas no emprego da luz como uma alternativa terapêutica opcional às convencionais, ou em conjunto com estas, no tratamento de enfermidades.

\section{Referências}

BROWN, G. C. Nitric oxide and mitochondrial respiration. Biochemica et Biophysica Acta, Amsterdam, v. 1411, n. 2/3, p. 351-369, 1999.

CONLAN, M. J.; RAPLEY, J. W.; COBB, C. M. Biostimulation of wound healing by low-energy irradiation. Journal of Clinical Periodontology, Copenhagen, v. 23, n. 5, p. 492-496 1996.

CORAZZA, A. V. Fotobiomodulação comparativa entre o LASER e o LED de baixa intensidade na angiogênese de feridas cutâneas de ratos. 2005 Dissertação (Mestrado em Bioengenharia) - Escola de Engenharia de São Carlos/ Faculdade de Engenharia de Ribeirão Preto/Instituto de Química de São Carlos, São Carlos: USPSCar, 2005.

FUBINI, S. Influenza della luce sulla respirazione del tessuto nervoso. Archivio per lê scienze mediche da G. Bizzozero, 3, 1879.

GORBACHE WICH, E. The Influence of Rays of Different Colors on the Growth and Development of Mammals. St. Petersburgh, 1882. Em russo.

JAVAN, A.; BENNET, W. R.; HERRIOT, D. R. Population inversion and continuous opitcal maser oscillation in a gas discharge containing a He-Ne mixture. Physical Review Letters, New York, v. 6, n. 1, p. 106-110, 1961.

KARU, T. I. Photobiological Fundamentals of LowPower Therapy. IEEE Journal of Quantum Electronics, New York, v. 23, n. 10, p. 1703-1717, 1987.

KARU, T. I. Molecular mechanism of the therapeutic effect of low-intensity laser radiation. Life Sciences, Oxford, v. 2, n. 1, p. 53-74, 1988.

KARU, T. I. Photobiology of Lower-Power Laser Therapy. London: Harwood Academic, 1989.
KARU, T. I. Low-Power Laser Therapy. In: VODINH, T. (Ed.). Biomedical photonics handbook. Boca Raton: CRC Press, 2003. p. 1-25. cap. 48.

KARU, T. I.; ANDREICHUK, T.; RUABYKH, T. Changes in oxidative metabolism of murine splenn following diode laser $(660-950 \mathrm{~nm})$ irradiation: effect of cellular composition and radiation parameters. Lasers in Surgery and Medicine, New York, v. 13, n. 4, p. 453-462, 1993.

KARU, T. I.; KALENDO, G. S.; LETOKHOV, V. S.; LOBKO, V. V. Biostimulation of HeLa cells by low intensity visible light. Nuovo Cimento D, Bologna, v. 1, n. 6, p. 828-840, 1982a.

KARU, T. I.; KALENDO, G. S.; LETOKHOV, V. S.; LOBKO, V. V. Biological action of low-intensity visible light on HeLa cells as a function of the coherence, dose, wavelength, and irradiation dose. Soviet Journal of Quantum Electronics, New York, v. 13, n. 9, p. 11691172, 1983a.

KARU, T. I.; KALENDO, G. S.; LETOKHOV, V. S.; LOBKO, V. V. Biological action of low-intensity visible light on HeLa cells as a function of the coherence, dose, wavelength and irradiation regime. II. Soviet Journal of Quantum Electronics, New York, v. 13, n. 9, p. 1169$1172,1983 \mathrm{~b}$.

KARU, T. I.; TIPHLOVA, O. A.; LETOKHOV, V. S.; LOBKO, V. V. Stimulation of $E$. coli growth by laser and incoherent red light. Nuovo Cimento D, Bologna, v. 2, n. 4, p. 1138-1144, 1983c.

KARU, T. I.; KOLYAKOV, S. F. Exact Action Spectra for Cellular Responses Relevant to Phototherapy. Photomedicine and Laser Surgery, Larchmont, v. 23, n. 4, p. 355-361, 2005.

KARU, T. I.; TIPHLOVA, O. A.; FEDOSEYEVA, G. E.; KALENDO, G. S.; LETOKHOV, V. S.; LOBKO, V. V.; LYAPUNOVA, T. S.; POMOSHNIKOVA, N. A.; MEISSEL, M. N. Bioestimulation action of low-intensity monochromatic visible light: it is possible ? Laser Chemistry, New York, v. 5, n. 1, p. 19-25, 1984.

KARU, T. I.; TIPHLOVA, O. A.; MATVEYETS, Y. A.; YARTSEV., A. P.; LETOKHOV, V. S. Comparison of the effects of visible femtosecond laser pulses and continuous wave laser radiation of low average intensity on the clonogenecity of Escherichia coli. Journal of Photochemistry Photobiology B: Biology, Lausanne, v. 10, n. 4, p. 339-344, 1991.

KÜSTNER, H. Die biologische Wirkung von Strahlen verschiedenen Wellenlängen. Zentralblatt fur Gyndkologie, Jena, v. 55, p. 2986-X, 1931. 
MAIMANN, T. H. Patente n: 3,353,115 Ruby Laser Systems, Hughes Aircraft Laboratories, depositada no EUA em julho de 1960.

MARQUES, C. R. S. Análise Evolutiva da Cicatrização em Úlceras: LEDterapia e Oxigenoterapia Hiperbárica. 2004. Dissertação (Mestrado em Engenharia Biomédica) - Universidade do vale do Paraíba, São José dos Campos, 2004.

MEDICAL COLLEGE OF WINSCONSIN - MCW. Research. Disponível em: $<$ http://www.mcw.edu/ MCWHome.htm $>$. Acesso em 27 Out. 2008.

MESTER, E.; ESPIRY, T.; SZENDE, B.; TOTA, J. G. Effect of laser rays on wound healing. American Journal of Surgery, New York, v. 122, n. 4, p. 532-535, 1971.

MESTER, E.; SZENDE, B.; SPIRY, T.; SCHER, A. Laser stimulation of wound healing by laser rays Acta Chirurgica Academiae Scientiarum Hungaricae, Budapest, v. 13, n. 3, p. 315-324, 1972.

NASA Light Emitting Diode Homepage. Disponível em: $\quad<$ http://www.nasa.gov/home/hqnews/2003/nov/ HQ 03366 clinical trials.html $>$. Acesso em 27 Out. 2008.

POLIAKOV, K. L.; MARGOLIN, G. M.; FEDDER, V. L. La modification de la chronoxie (N. ishiadici) d'une grenouille a l'action sur son corps des rayons lumineux d'une différente longueur d'onde. The Journal of Physiology, London, (URSS), n. 18, p. 1012, 1935.

PONZA, G. De l'influence de la lumière colorée dans lê traitment de la folie. Annales Medico-Psychologique, Paris, v. 15, ser. 5, 1876.

SCHUBERT, E. F. Light Emitting Diodes. 2. ed. Cambridge: Cambridge Univestity Press, 2006.

SCHNETZLER, J. S. De l'influence de la lumière sur lê développment des larves des grenouilles. Archives des sciences Physiques et naturelles, Geneva, v. 51, p. $247-$ 258, 1874.

TAKAHASHI, Y.; HITOMI, S.; HIRATA, T.; FUKUSE, T.; YAMAZAKI, F.; CHO, K.; WADA, H.
Neovascularization effect with $\mathrm{He}-\mathrm{Ne}$ laser in the rat trachea. The Journal of Thoracic Cardiovascular Surgery, St. Louis, v. 40, n. 5, p. 288-291, 1992.

VERDONK, G. L'antagonisme biologique in vitro entre differents bandes visible du spectre solaire. Compte Rendus des Séances de la Société de Biologie, Paris, v. 124, p. 258-261, 1937.

VINCK, E.; CAGNIE, B. J.; CORNELISSEN, M. J.; DECLERCQ, H. A.; CAMBIER, D. C. Increased fibroblast proliferation induced by light emitting diode and low power laser irradiations. Lasers in Medical Science, London, v. 18, n. 2, p. 95-99, 2003.

VINCK, E.; COOREVITS, P.; CAGNIE， B. J.; MARTINE, M.; VANDERSTRAETEN, G.; CAMBIER, D. C. Evidence of changes in sural nerve condiction mediated by light emitting diode irradiation. Lasers in Medical Science, London, v. 20, n. 1, p. 35-40, 2005.

VO-DINH, T. Biomedical Photonics Handbook. Boca Raton: CRC Press LLC, 2003.

WHELAN, H. T.; BUCHMANN, E. V.; WHELAN, N. T.; TURNER, S. G.; CEVENINI, V.; STINSON, H.; IGNATIUS, R.; MARTIN, T.; CWIKLINSKI, J.; MEYER, G. A.; HODGSON, B.; GOULD, L.; KANE, M.; CHEN, G.; CAVINESS, J.; NASA Light Emitting Diode Medical Applications From Deep Space to Deep Sea. Space Technology and Applications International Forum-2001.

American Institute of Physics, New York, p. 35-45, 2001.

YU, W.; NAIM, J, A.; LANZAFAME, R. J. Effects of photostimulation on wound healing in diabetic mice. Laser in Surgery and medicine, New York, v. 29, n. 2, p. 105-106, 1997.

YU, W.; NAIM, J. A.; MCGOWAN, M; IPPOLITO, K.; LANZAFAME, R. J. Photomodulation of oxidative metabolism and electron chain enzymes in rat liver mitochondria. Photochemistry and Photobiology, Oxford, v. 66, n. 6, p. 866-871, 1997. 